\title{
A note on the proofs of generalized Radon inequality
}

\author{
Yongtao Li, Xian-Ming GU*, Jianci XiaO
}

\begin{abstract}
In this paper, we introduce and prove several generalizations of the Radon inequality. The proofs in the current paper unify and also are simpler than those in early published work. Meanwhile, we find and show the mathematical equivalences among the Bernoulli inequality, the weighted AM-GM inequality, the Hölder inequality, the weighted power mean inequality and the Minkowski inequality. Finally, some applications involving the results proposed in this work are shown.
\end{abstract}

\section{INTRODUCTION}

The well-known Bergström inequality (see e.g. [1-3]) says that if $x_{k}, y_{k}$ are real numbers and $y_{k}>0$ for $1 \leq k \leq n$, then

$$
\frac{x_{1}^{2}}{y_{1}}+\frac{x_{2}^{2}}{y_{2}}+\cdots+\frac{x_{n}^{2}}{y_{n}} \geq \frac{\left(x_{1}+x_{2}+\cdots+x_{n}\right)^{2}}{y_{1}+y_{2}+\cdots+y_{n}}
$$

and the equality holds if and only if $\frac{x_{1}}{y_{1}}=\frac{x_{2}}{y_{2}}=\cdots=\frac{x_{n}}{y_{n}}$.

Some generalizations of the inequality (1) can be found in $[4,5]$. Actually, the following Radon inequality (2) is just a direct consequence: If $b_{1}, b_{2}, \ldots, b_{n}$ are positive real numbers and $a_{1}, a_{2}, \ldots, a_{n}, m$ are nonnegative real numbers, then

$$
\frac{a_{1}^{m+1}}{b_{1}^{m}}+\frac{a_{2}^{m+1}}{b_{2}^{m}}+\cdots+\frac{a_{n}^{m+1}}{b_{n}^{m}} \geq \frac{\left(a_{1}+a_{2}+\cdots+a_{n}\right)^{m+1}}{\left(b_{1}+b_{2}+\cdots+b_{n}\right)^{m}} .
$$

When $m=1,(2)$ reduces to (1). For more details on the Radon inequality (2), the readers can refer to [6, pp. 1351] and $[7,8,10]$. In fact, it is not hard to prove that (1) is equivalent to the Cauchy-Buniakovski-Schwarz inequality (see [9, pp. 34-35, Theorem 1.6.1]) stated as follows: if $a_{1}, \ldots, a_{n}, b_{1}, \ldots, b_{n}$

2010 Mathematics Subject Classification. Primary: 26D15.

Key words and phrases. The Bergström inequality, the Radon inequality, the weighted power mean inequality, equivalence, the Hölder inequality.

Full paper. Received 9 July 2018, revised 3 August 2018, accepted 5 September 2018, available online 15 December 2018.

$*$ Corresponding author. 
are nonnegative real numbers, then

$$
\sum_{k=1}^{n} a_{k} \sum_{k=1}^{n} b_{k} \geq\left(\sum_{k=1}^{n} \sqrt{a_{k} b_{k}}\right)^{2}
$$

In [14, Theorem 1], Yang has given a generalization of the Radon inequality as follows: if $a_{1}, a_{2}, \ldots, a_{n}$ are nonnegative real numbers and $b_{1}, b_{2}, \ldots, b_{n}$ are positive real numbers, then for $r \geq 0, s \geq 0$ and $r \geq s+1$,

$$
\frac{a_{1}^{r}}{b_{1}^{s}}+\frac{a_{2}^{r}}{b_{2}^{s}}+\cdots+\frac{a_{n}^{r}}{b_{n}^{s}} \geq \frac{\left(a_{1}+a_{2}+\cdots+a_{n}\right)^{r}}{n^{r-s-1}\left(b_{1}+b_{2}+\cdots+b_{n}\right)^{s}} .
$$

The weighted power mean inequality (refer to [12, pp. 111-112, Theorem 10.5], [7, pp. 12-15] and [13] for details) is defined as follows: if $x_{1}, x_{2}, \ldots, x_{n}$ are nonnegative real numbers and $p_{1}, p_{2}, \ldots, p_{n}$ are positive real numbers, then for $r \geq s>0$, we have

$$
\left(\frac{p_{1} x_{1}^{r}+p_{2} x_{2}^{r}+\cdots+p_{n} x_{n}^{r}}{p_{1}+p_{2}+\cdots+p_{n}}\right)^{\frac{1}{r}} \geq\left(\frac{p_{1} x_{1}^{s}+p_{2} x_{2}^{s}+\cdots+p_{n} x_{n}^{s}}{p_{1}+p_{2}+\cdots+p_{n}}\right)^{\frac{1}{s}} .
$$

In the present paper, we give three concise proofs and some applications of the generalized Radon inequality (3), and then present equivalence relations between the weighted power mean inequality and the Radon inequality. Furthermore, we summarize the equivalences among the weighted AM-GM inequality, the Hölder inequality, the weighted power mean inequality and the Minkovski inequality.

\section{MAIN RESUltS}

In this section, we first give three different and concise methods for proving the generalized Radon inequality (3). To read for convenience, the result obtained by Yang [14] can be cited as the following theorem.

Theorem 2.1. If $a_{1}, a_{2}, \ldots, a_{n}$ are nonnegative real numbers and $b_{1}, b_{2}, \ldots, b_{n}$ are positive real numbers, then for $s \geq 0$ and $r \geq s+1$,

$$
\frac{a_{1}^{r}}{b_{1}^{s}}+\frac{a_{2}^{r}}{b_{2}^{s}}+\cdots+\frac{a_{n}^{r}}{b_{n}^{s}} \geq \frac{\left(a_{1}+a_{2}+\cdots+a_{n}\right)^{r}}{n^{r-s-1}\left(b_{1}+b_{2}+\cdots+b_{n}\right)^{s}} .
$$

Proof 1. By using the Radon inequality (2), we have

$$
\sum_{k=1}^{n} \frac{a_{k}^{r}}{b_{k}^{s}}=\sum_{k=1}^{n} \frac{\left(a_{k}^{\frac{r}{s+1}}\right)^{s+1}}{b_{k}^{s}} \geq \frac{\left(a_{1}^{\frac{r}{s+1}}+a_{2}^{\frac{r}{s+1}}+\cdots+a_{n}^{\frac{r}{s+1}}\right)^{s+1}}{\left(b_{1}+b_{2}+\cdots+b_{n}\right)^{s}}
$$

Note that $r \geq s+1 \geq 1$, then $\frac{r}{s+1}-1 \geq 0$. Using the Radon inequality again, it follows that

$$
\sum_{k=1}^{n} a_{k}^{\frac{r}{s+1}}=\sum_{k=1}^{n} \frac{a_{k}^{\frac{r}{s+1}}}{1^{\frac{r}{s+1}-1}} \geq \frac{\left(a_{1}+a_{2}+\cdots+a_{n}\right)^{\frac{r}{s+1}}}{(1+1+\cdots+1)^{\frac{r}{s+1}}-1}
$$


According to inequalities (6) and (7), we clearly have

$$
\frac{a_{1}^{r}}{b_{1}^{s}}+\frac{a_{2}^{r}}{b_{2}^{s}}+\cdots+\frac{a_{n}^{r}}{b_{n}^{s}} \geq \frac{\left(a_{1}+a_{2}+\cdots+a_{n}\right)^{r}}{n^{r-s-1}\left(b_{1}+b_{2}+\cdots+b_{n}\right)^{s}} .
$$

Therefore, the desired result (5) is obtained.

Proof 2. Let the concave function $f:(0,+\infty) \rightarrow \mathbb{R}$ be $f(x)=\ln x$. We observe that the weighted Jensen inequality: for $q_{1}, q_{2}, q_{3} \in[0,1]$ with $q_{1}+$ $q_{2}+q_{3}=1$ and positive real numbers $x_{1}, x_{2}, x_{3}$, then we have

$$
q_{1} f\left(x_{1}\right)+q_{2} f\left(x_{2}\right)+q_{3} f\left(x_{3}\right) \leq f\left(q_{1} x_{1}+q_{2} x_{2}+q_{3} x_{3}\right),
$$

and the equality holds if and only if $x_{1}=x_{2}=x_{3}$. We denote

$$
U_{n}(a)=\left(\frac{a_{1}^{r}}{b_{1}^{s}}+\frac{a_{2}^{r}}{b_{2}^{s}}+\cdots+\frac{a_{n}^{r}}{b_{n}^{s}}\right)^{-1}
$$

and

$$
H_{n}(b)=\left(b_{1}+b_{2}+\cdots+b_{n}\right)^{-1} .
$$

Consider $x_{1}=\frac{a_{k}^{r}}{b_{k}^{s}} U_{n}(a), x_{2}=b_{k} H_{n}(b), x_{3}=\frac{1}{n}$ and $q_{1}=\frac{1}{r}, q_{2}=\frac{s}{r}, q_{3}=$ $\frac{r-s-1}{r}$ (observe that $q_{3} \geq 0$ from $r \geq s+1$ ). Thus we have

$$
\begin{aligned}
& a_{k}\left(U_{n}(a)\right)^{\frac{1}{r}} \cdot\left(H_{n}(b)\right)^{\frac{s}{r}} \cdot\left(\frac{1}{n}\right)^{\frac{r-s-1}{r}} \\
& \leq \frac{1}{r} \cdot \frac{a_{k}^{r}}{b_{k}^{s}} U_{n}(a)+\frac{s}{r} \cdot b_{k} H_{n}(b)+\frac{r-s-1}{r} \cdot \frac{1}{n} .
\end{aligned}
$$

Summing up over $k(k=1,2, \ldots, n)$, we obtain

$$
\begin{aligned}
& \sum_{k=1}^{n} a_{k}\left(U_{n}(a)\right)^{\frac{1}{r}} \cdot\left(H_{n}(b)\right)^{\frac{s}{r}} \cdot\left(\frac{1}{n}\right)^{\frac{r-s-1}{r}} \\
& \leq \sum_{k=1}^{n}\left(\frac{1}{r} \cdot \frac{a_{k}^{r}}{b_{k}^{s}} U_{n}(a)+\frac{s}{r} \cdot b_{k} H_{n}(b)+\frac{r-s-1}{r} \cdot \frac{1}{n}\right)=1 .
\end{aligned}
$$

The required inequality (5) follows.

For many numerical inequalities, the induction is sometimes a useful method used to establish a given statement for all natural numbers. We now give the third proof of Theorem 2.1 by mathematical induction. To state this proof clearly, let us start with the following lemma.

Lemma 2.1. If $a_{1}, a_{2}, \ldots, a_{n}, b_{1}, b_{2}, \ldots, b_{n}$ are nonnegative real numbers and $\lambda_{1}, \lambda_{2}, \ldots, \lambda_{n}$ are nonnegative real numbers such that $\lambda_{1}+\lambda_{2}+\cdots+\lambda_{n}=$ 1 , then

$$
\prod_{k=1}^{n} a_{k}^{\lambda_{k}}+\prod_{k=1}^{n} b_{k}^{\lambda_{k}} \leq \prod_{k=1}^{n}\left(a_{k}+b_{k}\right)^{\lambda_{k}}
$$


Proof of Lemma 2.1. According to the weighted AM-GM inequality, we have

$$
\prod_{k=1}^{n}\left(\frac{a_{k}}{a_{k}+b_{k}}\right)^{\lambda_{k}} \leq \sum_{k=1}^{n} \lambda_{k}\left(\frac{a_{k}}{a_{k}+b_{k}}\right) .
$$

Similarly, we get

$$
\prod_{k=1}^{n}\left(\frac{b_{k}}{a_{k}+b_{k}}\right)^{\lambda_{k}} \leq \sum_{k=1}^{n} \lambda_{k}\left(\frac{b_{k}}{a_{k}+b_{k}}\right) .
$$

Summing up these two inequalities, it holds

$$
\prod_{k=1}^{n} \frac{1}{\left(a_{k}+b_{k}\right)^{\lambda_{k}}}\left[\prod_{k=1}^{n} a_{k}^{\lambda_{k}}+\prod_{k=1}^{n} b_{k}^{\lambda_{k}}\right] \leq \sum_{k=1}^{n} \lambda_{k}=1,
$$

which leads to the desired result (8).

Remark 2.1. A particular case $b_{1}=b_{2}=\cdots=b_{n}=1, \lambda_{1}=\lambda_{2}=\cdots=$ $\lambda_{n}=\frac{1}{n}$ in (8) yields

$$
\left(1+a_{1}\right)\left(1+a_{2}\right) \cdots\left(1+a_{n}\right) \geq\left[1+\left(a_{1} a_{2} \cdots a_{n}\right)^{\frac{1}{n}}\right]^{n},
$$

which is a famous inequality, called the Chrystal inequality (refer to [7, pp. 61]), so Lemma 2.1 can be regarded as a generalization of the Chrystal inequality.

Proof 3. Use the induction on $n \in \mathbb{N}^{+}$. When $n=1$, the result is obviously obtained. Assume that (5) is true for $n=m$, that is

$$
\frac{a_{1}^{r}}{b_{1}^{s}}+\frac{a_{2}^{r}}{b_{2}^{s}}+\cdots+\frac{a_{m}^{r}}{b_{m}^{s}} \geq \frac{\left(a_{1}+a_{2}+\cdots+a_{m}\right)^{r}}{m^{r-s-1}\left(b_{1}+b_{2}+\cdots+b_{m}\right)^{s}} .
$$

When $n=m+1$, we need to prove the following inequality:

$$
\begin{aligned}
\sum_{k=1}^{m+1} \frac{a_{k}^{r}}{b_{k}^{s}} & =\sum_{k=1}^{m} \frac{a_{k}^{r}}{b_{k}^{s}}+\frac{a_{m+1}^{r}}{b_{m+1}^{s}} \\
& \geq \frac{\left(a_{1}+a_{2}+\cdots+a_{m}\right)^{r}}{m^{r-s-1}\left(b_{1}+b_{2}+\cdots+b_{m}\right)^{s}}+\frac{a_{m+1}^{r}}{b_{m+1}^{s}} \text { (by induction assumption) } \\
& =\frac{\left[\left(R_{m}(a)+\frac{a_{m+1}^{r}}{b_{m+1}^{s}}\right)^{\frac{1}{r}}\left(S_{m}(b)+b_{m+1}\right)^{\frac{s}{r}}(m+1)^{\frac{r-s-1}{r}}\right]^{r}}{(m+1)^{r-s-1}\left(S_{m}(b)+b_{m+1}\right)^{s}} \\
& \geq \frac{\left[\left(R_{m}(a)\right)^{\frac{1}{r}}\left(S_{m}(b)\right)^{\frac{s}{r}} m^{\frac{r-s-1}{r}}+\left(\frac{a_{m+1}^{r}}{b_{m+1}^{s}}\right)^{\frac{1}{r}} b_{m+1}^{\frac{s}{r}} 1^{\frac{r-s-1}{r}}\right]^{r}}{(m+1)^{r-s-1}\left(b_{1}+\cdots+b_{m}+b_{m+1}\right)^{s}}
\end{aligned}
$$

(by a special case $n=3$ in (8))

$$
=\frac{\left(a_{1}+\cdots+a_{m}+a_{m+1}\right)^{r}}{(m+1)^{r-s-1}\left(b_{1}+\cdots+b_{m}+b_{m+1}\right)^{s}},
$$


where $R_{m}(a)=\frac{\left(a_{1}+\cdots+a_{m}\right)^{r}}{m^{r-s-1}\left(b_{1}+\cdots+b_{m}\right)^{s}}$ and $S_{m}(b)=b_{1}+b_{2}+\cdots+b_{m}$. Thus, the inequality (5) holds for $n=m+1$, so the proof of the induction step is completed.

In the next theorem, we will prove the equivalence relations between the weighted power mean inequality and the Radon inequality, which is partly motivated by a slight observation of the inequality (7).

Theorem 2.2. The Radon inequality (2) is equivalent to the weighted power mean inequality (4).

Proof. $\Rightarrow$ By the Radon inequality (2) and $y_{1}, y_{2}, \ldots, y_{n} \in[0,+\infty)$, we have

$$
\begin{aligned}
p_{1} y_{1}^{\frac{r}{s}}+p_{2} y_{2}^{\frac{r}{s}}+\cdots+p_{n} y_{n}^{\frac{r}{s}} & =\frac{\left(p_{1} y_{1}\right)^{\frac{r}{s}}}{p_{1}^{\frac{r}{s}-1}}+\frac{\left(p_{2} y_{2}\right)^{\frac{r}{s}}}{p_{2}^{\frac{r}{s}}-1}+\cdots+\frac{\left(p_{n} y_{n}\right)^{\frac{r}{s}}}{p_{n}^{\frac{r}{s}-1}} \\
& \geq \frac{\left(p_{1} y_{1}+p_{2} y_{2}+\cdots+p_{n} y_{n}\right)^{\frac{r}{s}}}{\left(p_{1}+p_{2}+\cdots+p_{n}\right)^{\frac{r}{s}-1}},
\end{aligned}
$$

which means that

$$
\frac{p_{1} y_{1}^{\frac{r}{s}}+p_{2} y_{2}^{\frac{r}{s}}+\cdots+p_{n} y_{n}^{\frac{r}{s}}}{p_{1}+p_{2}+\cdots+p_{n}} \geq\left(\frac{p_{1} y_{1}+p_{2} y_{2}+\cdots+p_{n} y_{n}}{p_{1}+p_{2}+\cdots+p_{n}}\right)^{\frac{r}{s}} .
$$

Let $y_{k}=x_{k}^{s}$ for all $x_{k} \geq 0(k=1,2, \ldots, n)$ in $(9)$. Thus, we can obtain the following weighted power mean inequality (4)

$$
\left(\frac{p_{1} x_{1}^{r}+p_{2} x_{2}^{r}+\cdots+p_{n} x_{n}^{r}}{p_{1}+p_{2}+\cdots+p_{n}}\right)^{\frac{1}{r}} \geq\left(\frac{p_{1} x_{1}^{s}+p_{2} x_{2}^{s}+\cdots+p_{n} x_{n}^{s}}{p_{1}+p_{2}+\cdots+p_{n}}\right)^{\frac{1}{s}} .
$$

$\Leftarrow$ Let $p_{k}=b_{k}, x_{k}=\frac{a_{k}}{b_{k}}$ and $r=m+1(m \geq 0), s=1$ in (4). Then, we have $\left[\frac{1}{b_{1}+b_{2}+\cdots+b_{n}}\left(\frac{a_{1}^{m+1}}{b_{1}^{m}}+\frac{a_{2}^{m+1}}{b_{2}^{m}}+\cdots+\frac{a_{n}^{m+1}}{b_{n}^{m}}\right)\right]^{\frac{1}{m+1}} \geq \frac{a_{1}+a_{2}+\cdots+a_{n}}{b_{1}+b_{2}+\cdots+b_{n}}$,

which implies that the Radon inequality (2) is achieved.

Theorem 2.3. The following inequalities are mutually equivalent:

(i) The Bernoulli inequality;

(ii) The weighted AM-GM inequality;

(iii) The Hölder inequality;

(iv) The weighted power mean inequality;

(v) The Minkovski inequality;

(vi) The Radon inequality.

Proof. The equivalence between (iv) and (vi) is given in Theorem 2.2, the equivalence among (i), (iii) and (vi), one can find in [11] as well as (ii), (iii) and (iv) in [15], the equivalence between (iii) and (v) is shown in [16]. 
Corollary 2.1. If $a_{1}, a_{2}, \ldots, a_{n}, b_{1}, b_{2}, \ldots, b_{n}$ are positive real numbers, then for $m \leq-1$, the following inequality holds

$$
\frac{a_{1}^{m+1}}{b_{1}^{m}}+\frac{a_{2}^{m+1}}{b_{2}^{m}}+\cdots+\frac{a_{n}^{m+1}}{b_{n}^{m}} \geq \frac{\left(a_{1}+a_{2}+\cdots+a_{n}\right)^{m+1}}{\left(b_{1}+b_{2}+\cdots+b_{n}\right)^{m}} .
$$

Proof. Since $m \leq-1$, thus by the inequality (2), we have

$$
\begin{aligned}
\frac{a_{1}^{m+1}}{b_{1}^{m}}+\frac{a_{2}^{m+1}}{b_{2}^{m}}+\cdots+\frac{a_{n}^{m+1}}{b_{n}^{m}} & =\frac{b_{1}^{-m}}{a_{1}^{-m-1}}+\frac{b_{2}^{-m}}{a_{2}^{-m-1}}+\cdots+\frac{b_{n}^{-m}}{a_{n}^{-m-1}} \\
& \geq \frac{\left(b_{1}+b_{2}+\cdots+b_{n}\right)^{-m}}{\left(a_{1}+a_{2}+\cdots+a_{n}\right)^{-m-1}} .
\end{aligned}
$$

Therefore, the inequality (10) holds.

Corollary 2.2. If $a_{1}, a_{2}, \ldots, a_{n}, b_{1}, b_{2}, \ldots, b_{n}$ are positive real numbers, then for nonpositive real numbers $r, s$ such that $r \geq s+1$, we have

$$
\frac{a_{1}^{r}}{b_{1}^{s}}+\frac{a_{2}^{r}}{b_{2}^{s}}+\cdots+\frac{a_{n}^{r}}{b_{n}^{s}} \geq \frac{\left(a_{1}+a_{2}+\cdots+a_{n}\right)^{r}}{n^{r-s-1}\left(b_{1}+b_{2}+\cdots+b_{n}\right)^{s}} .
$$

Proof. For $r \leq 0$ and $s \leq 0$, the inequalities $-s \geq-r+1,-r \geq 0,-s \geq 0$ hold. By the inequality (5), we obtain

$$
\begin{aligned}
\frac{a_{1}^{r}}{b_{1}^{s}}+\frac{a_{2}^{r}}{b_{2}^{s}}+\cdots+\frac{a_{n}^{r}}{b_{n}^{s}} & =\frac{b_{1}^{-s}}{a_{1}^{-r}}+\frac{b_{2}^{-s}}{a_{2}^{-r}}+\cdots+\frac{b_{n}^{-s}}{a_{n}^{-r}} \\
& \geq \frac{\left(b_{1}+b_{2}+\cdots+b_{n}\right)^{-s}}{n^{-s-(-r)-1}\left(a_{1}+a_{2}+\cdots+a_{n}\right)^{-r}} \\
& =\frac{\left(a_{1}+a_{2}+\cdots+a_{n}\right)^{r}}{n^{r-s-1}\left(b_{1}+b_{2}+\cdots+b_{n}\right)^{s}} .
\end{aligned}
$$

So, the inequality (11) holds.

Corollary 2.3. If $a_{1}, a_{2}, \ldots, a_{n}, c_{1}, c_{2}, \ldots, c_{n}$ are positive real numbers, and $m$ is real numbers such that $m>0$ or $m \leq-1$, then

$$
\frac{a_{1}}{c_{1}}+\frac{a_{2}}{c_{2}}+\cdots+\frac{a_{n}}{c_{n}} \geq \frac{\left(a_{1}+a_{2}+\cdots+a_{n}\right)^{m+1}}{\left(a_{1} c_{1}^{\frac{1}{m}}+a_{2} c_{2}^{\frac{1}{m}}+\cdots+a_{n} c_{n}^{\frac{1}{m}}\right)^{m}} .
$$

Proof. Consider $b_{k}=a_{k} c_{k}^{\frac{1}{m}}$ for all $1 \leq k \leq n$ in the inequality (2) and (10). Thus, we obtain the inequality (12).

Corollary 2.4. If $a, b \in \mathbb{R}, a<b, m \geq 0$ or $m \leq-1, f, g:[a, b] \rightarrow(0,+\infty)$ are integrable functions on $[a, b]$ for all $x \in[a, b]$, then

$$
\int_{a}^{b} \frac{(f(x))^{m+1}}{(g(x))^{m}} \mathrm{~d} x \geq \frac{\left(\int_{a}^{b} f(x) \mathrm{d} x\right)^{m+1}}{\left(\int_{a}^{b} g(x) \mathrm{d} x\right)^{m}} .
$$


Proof. Let $n \in \mathbb{N}_{+}, x_{k}=a+k \frac{b-a}{n}, k \in\{0,1, \ldots, n\}$ and $\xi_{k} \in\left[x_{k-1}, x_{k}\right]$. By using the inequalities (2) and (10), it follows

$$
\sum_{k=1}^{n} \frac{\left(f\left(\xi_{k}\right)\right)^{m+1}}{\left(g\left(\xi_{k}\right)\right)^{m}} \geq \frac{\left(\sum_{k=1}^{n} f\left(\xi_{k}\right)\right)^{m+1}}{\left(\sum_{k=1}^{n} g\left(\xi_{k}\right)\right)^{m}} .
$$

It holds that

$$
\sigma\left(\frac{(f(x))^{m+1}}{(g(x))^{m}}, \Delta_{n}, \xi_{k}\right) \geq \frac{\left[\sigma\left(f(x), \Delta_{n}, \xi_{k}\right)\right]^{m+1}}{\left[\sigma\left(g(x), \Delta_{n}, \xi_{k}\right)\right]^{m}},
$$

where $\sigma\left(f(x), \Delta_{n}, \xi_{k}\right)$ is the corresponding Riemann sum of $f(x)$, of $\Delta_{n}=$ $\left(x_{0}, x_{1}, \ldots, x_{n}\right)$ division and the intermediate $\xi_{k}$ points. By passing to limit in inequality above, when $n$ tends to infinity, the inequality(13) follows.

Corollary 2.5. If $a, b \in \mathbb{R}, a<b, r s \geq 0, r \geq s+1, f, g:[a, b] \rightarrow(0,+\infty)$ are integrable functions on $[a, b]$ for any $x \in[a, b]$, then

$$
\int_{a}^{b} \frac{(f(x))^{r}}{(g(x))^{s}} \mathrm{~d} x \geq \frac{\left(\int_{a}^{b} f(x) \mathrm{d} x\right)^{r}}{(b-a)^{r-s-1}\left(\int_{a}^{b} g(x) \mathrm{d} x\right)^{s}} .
$$

Proof. Since the conclusion can be obtained via using the same method of Corollary 2.4, we omit the details here.

Proposition 2.1. If $a, b, c$ are the lengths of the sides of a triangle and $2 S=a+b+c$, then

$$
\frac{a^{n}}{b+c}+\frac{b^{n}}{c+a}+\frac{c^{n}}{a+b} \geq\left(\frac{2}{3}\right)^{n-2} S^{n-1}, \quad n \geq 1 .
$$

Proof. When $n=1$, the result (14) equals to the Nesbitt inequality (see [9, p. 16, Example 1.4.8] or [12, p. 2, Exercise 1.3]). For $n \geq 2$, we obtain

$$
\begin{aligned}
\frac{a^{n}}{b+c}+\frac{b^{n}}{c+a}+\frac{c^{n}}{a+b} & \geq \frac{(a+b+c)^{n}}{3^{n-1-1}(b+c+c+a+a+b)} \\
& =\left(\frac{2}{3}\right)^{n-2} S^{n-1},
\end{aligned}
$$

by using the inequality (5).

Proposition 2.2. Let $a_{1}, a_{2}, \ldots, a_{n}$ be positive real numbers such that $a_{1}+$ $a_{2}+\cdots+a_{n}=s$ and $p \geqslant q+1 \geqslant 1$. Then

$$
\sum_{k=1}^{n} \frac{a_{k}^{p}}{\left(s-a_{k}\right)^{q}} \geq \frac{s^{p-q}}{(n-1)^{q} n^{p-q-1}} .
$$

Proof. By the inequality (5), the inequality above is easily obtained. 
Proposition 2.3. Let $x, y$, and $z$ be positive numbers with $x y z=1$. Then

$$
\frac{x^{3}}{(1+y)(1+z)}+\frac{y^{3}}{(1+z)(1+x)}+\frac{z^{3}}{(1+x)(1+y)} \geq \frac{3}{4} \text {. }
$$

Proof. By using the generalized Radon inequality (5), we obtain

$$
\begin{aligned}
& \frac{x^{3}}{(1+y)(1+z)}+\frac{y^{3}}{(1+z)(1+x)}+\frac{z^{3}}{(1+x)(1+y)} \\
\geq & \frac{(x+y+z)^{3}}{3((1+y)(1+z)+(1+z)(1+x)+(1+x)(1+y))} \\
= & \frac{(x+y+z)^{3}}{9+6(x+y+z)+3(x y+y z+z x)} \\
& \left(\text { by a general inequality } 3(x y+y z+z x) \leq(x+y+z)^{2}\right) \\
\geq & \frac{(x+y+z)^{3}}{9+6(x+y+z)+(x+y+z)^{2}} .
\end{aligned}
$$

Since $x+y+z \geq 3 \sqrt[3]{x y z}=3$, it is not hard to prove that $\frac{(x+y+z)^{3}}{9+6(x+y+z)+(x+y+z)^{2}}$ $\geq \frac{3}{4}$. By the way, another proof can be found in [9, pp. 139-140].

\section{REFERENCES}

[1] K. Y. Fan, Generalization of Bergsrröm inequality, American Mathematical Monthly, 66 (2) (1959), 153-154.

[2] H. Bergström, A triangle inequality for matrices, Den Elfte Skandinaviske Matematikerkongress, Trondheim, 1949, Johan Grundt Tanums Forlag, Oslo, 1952, 264-267.

[3] R. Bellman, Notes on matrix theory-IV (An inequality due to Bergström), American Mathematical Monthly, 62 (3) (1955), 172-173.

[4] S. Abramovich, B. Mond, J. E. Pečarić, Sharpening Jensen's inequality and a majorization theorem, Journal of Mathematical Analysis and Applications, 214 (2) (1997), 721-728.

[5] J. E. Pečarić, F. Proschan, Y. I. Tong, Convex Functions, Partial Orderings, and Statistical Applications, Mathematics in Science and Engineering, Academic Press, San Diego, CA, 1992.

[6] J. Radon, Über die absolut additiven Mengenfunktionen, Wiener Sitzungsber (IIa), 122 (1913), 1295-1438.

[7] G. H. Hardy, J. E. Littlewood, G. Pólya, Inequalities, Cambridge University Press, Cambridge, UK, 1934.

[8] C. B. Morrey, A class of representations of manifolds, American Mathematical Monthly, 55 (1) (1933), 683-707.

[9] R. B. Manfrino, J. A. G. Ortega, R. V. Delgado, Inequalities: A Mathematical Olympiad Approach, Birkhäuser, Basel-Boston-Berlin, 2009.

[10] C. Mortici, A new refinement of the Radon inequality, Mathematical Communications, 16 (2) (2011), 319-324. 
[11] D. M. Bătinetu-Giurgiu, O. T. Pop, A generalization of Radon's inequality, Creative Mathematics and Informatics, 19 (2) (2010), 116-121.

[12] Z. Cvetkovski, Inequalities. Theorems, Techniques and Selected Problems, SpringerVerlag Berlin Heidelberg, Heidelberg, 2012.

[13] D. S. Mitrinović, J. E. Pečarić, A. M. Fink, Classical and New Inequalities in Analysis, Mathematics and Its Applications (East European Series), Vol. 61, Kluwer Amcademic, Dordrecht, 1993.

[14] K.-C. Yang, A note and generalization of a fractional inequality, Journal of Yueyang Normal University (Natural Science Edition), 15 (4) (2002), 9-11. (in Chinese)

[15] Y. Li, X.-M. Gu, J. Zhao, The weighted arithmetic mean-geometric mean inequality is equivalent to the Hölder inequality, Symmetry, 10 (9) (2018), Article ID: 380, 5 pages.

[16] L. Maligranda, Equivalence of the Hölder-Rogers and Minkowski inequalities, Mathematical Inequalities \& Applications, 4 (2) (2011), 203-207.

\section{Yongtao Li}

School of Mathematics and Statistics

Central South University

Changsha, Hunan 410083

P.R. China

E-mail address: yli777@qq.com

\section{Xian-Ming Gu}

School of Economic Mathematics

Institute of MATHEMATiCS

Southwestern University of Finance And Economic

Chengdu, Sichuan 611731

P.R. China

E-mail address: guxianming@live.cn

guxm@swufe.edu.cn

\section{JiANCI XiaO}

School of Mathematical Sciences

ZHEJIANG UNIVERSITY

Hangzhou, Zhejiang 310027

P.R. China

E-mail address: jcxshaw@outlook.com 